AperTO - Archivio Istituzionale Open Access dell'Università di Torino

\title{
Computational modeling of the mechanics of hierarchical materials
}

\section{This is the author's manuscript}

Original Citation:

Availability:

This version is available http://hdl.handle.net/2318/1595143

since 2016-10-27T10:26:10Z

Published version:

DOI:10.1557/mrs.2016.185

Terms of use:

Open Access

Anyone can freely access the full text of works made available as "Open Access". Works made available under a Creative Commons license can be used according to the terms and conditions of said license. Use of all other works requires consent of the right holder (author or publisher) if not exempted from copyright protection by the applicable law. 
This is the author's final version of the contribution published as:

Signetti, Stefano; Bosia, Federico; Pugno, Nicola M.. Computational modeling of the mechanics of hierarchical materials. MRS BULLETIN. 41 (09) pp: 694-699.

DOI: $10.1557 / \mathrm{mrs} .2016 .185$

The publisher's version is available at:

http://www.journals.cambridge.org/abstract_S0883769416001858

When citing, please refer to the published version.

Link to this full text:

http://hdl.handle.net/2318/1595143 


\title{
Computational modeling of the mechanics of hierarchical materials
}

Stefano Signetti, Federico Bosia, and Nicola M. Pugno

\begin{abstract}
Structural hierarchy coupled with material heterogeneity is often identified in natural materials, from nano- to macroscale. It combines disparate mechanical properties, such as strength and toughness, and multifunctionality, such as smart adhesion, water repellence, self-cleaning, and self-healing. These architectures can be employed in synthetic bioinspired structured materials, also adopting constituents with superior mechanical properties, such as carbon nanotubes or graphene. Advanced computational modeling is essential to understand the complex mechanisms that couple material, structural, and topological hierarchy, merging phenomena of different nature, size, and time scales. Numerical modeling also allows extensive parametric studies for the optimization of material properties and arrangement, avoiding time-consuming and complex experimental trials and providing guidance in the fabrication of novel advanced materials. Here, we review some of the most promising approaches, with a focus on the methods developed by our group.
\end{abstract}

Keywords: simulation, fracture, composite, nanostructure, biological

\section{Introduction}

For centuries, Nature has provided inspiration for man in the design and manufacture of structural materials. Many natural materials display fascinating physical and mechanical properties that, until recently, have been hard to replicate in artificial materials and structures. These include high stiffness and low density, ${ }^{1}$ strong adhesion and easy detachment, ${ }^{2}$ self-sharpening, ${ }^{3}$ self-healing, growth, and adaptive tissue organization, water-repellence, self-cleaning, and super hydrophobic or super-hydrophilic behaviors. ${ }^{4,5}$ All of these properties are usually related to complex multiscale structural arrangements of different constituents, from the nano- to the macroscale, involving material mixing and grading, interaction between constituents, and, most importantly, hierarchy, 
intended as the property whereby a material exhibits structure on more than one length scale. ${ }^{6-8}$

In the study of bioinspired materials, the focus is on the link between material properties and the emerging of specific functions across all relevant scales. The main lesson from Nature is that complex behavior and functionality derives from structure, hierarchy, and optimal organization of simple basic components. This is in stark contrast to many engineered materials, which exploit the properties of exotic metals or polymers, with associated high production and environmental costs. Nature has successfully done more with less, in terms of material design and production. This paradigm could, in principle, also apply to synthetic materials, and can be transposed to many fields in engineering, with the potential to do even better than Nature, in the absence of limiting biological constraints.

Experimental tests and measurements on hierarchical materials can be difficult to rationalize due to high uncertainties and statistical dispersion at the nanoscale, difficulties in sample manipulation and characterization, problematic reproducibility of boundary conditions and, in some cases, the impossibility of direct experimental verification at all the different size scales. Thus, due to the complexity and cross-scale interaction of the physical phenomena involved, further progress requires comprehensive numerical modeling. The challenge is to integrate multiple length scales and physical phenomena within the same simulation framework, since in hierarchical structures, a piecewise understanding of individual parts cannot simply be assembled or superimposed: the whole is greater than the sum of the individual parts. ${ }^{9,10}$

\section{Numerical modeling of biological and bioinspired material properties}

Computational methods for modeling the mechanics of hierarchical materials need to span the various size and time scales of the problems involved. These can be divided schematically into three broad categories: (1) nanoscale-methods like density functional theory (DFT) or molecular dynamics (MD), to achieve characterization of the basic constituents of hierarchical composites; (2) micro/mesoscale - fiber bundle model (FBM), lattice-spring model (LSM), discrete and 
meshless methods to reconstruct the role of hierarchy and material mixing in characteristic mechanical properties of composites; and (3) macro-scale-finite element methods (FEM) and discrete or boundary element methods (DEM or BEM) to model complex mechanical problems at the continuum level for solids, and particle methods for fluid mechanics, based on Navier-Stokes or Boltzmann equations. Figure 1 depicts the overall scenario of these multiscale analysis techniques.

Computational methods can provide new insights in the comprehension of fracture mechanisms in heterogeneous/hierarchical/multiscale structures, beginning with the nanoscale. Various multiscale models have been developed to capture the mechanisms involved in the optimization of global material mechanical properties. ${ }^{11-13}$ One example is represented by the so-called fiber bundle models (FBM) ${ }^{14}$ which are particularly appropriate for the simulation of fibrous materials, often occurring in biomaterials. Here, material structure at a certain size scale is modeled as a network of fibers arranged in parallel (same level) and in series (different levels) subjected to uniaxial tension, with statistically Weibull-distributed yield and fracture strengths or strains. Usually, an equal-load-sharing hypothesis is adopted, ${ }^{14}$ whereby when fibers fracture, stresses are redistributed uniformly among the remaining fibers in the bundle. Multiphase media are modeled by assigning different mechanical properties the fibers of each bundle.

We introduced a hierarchical extension of the Hierarchical Fibre Bundle Model (HFBM), whereby the input mechanical behavior of a subvolume or "fiber" at a given hierarchical level is statistically inferred from the average output deriving from repeated simulations at the lower level, down to the lowest hierarchical level ${ }^{15}$ (Figure 2a). Results from this and other implementations of HFBM show that specific hierarchical organizations can lead to increased damage resistance (e.g., self-similar fiber reinforced matrix materials) ${ }^{16}$ or that the interaction between hierarchy and material heterogeneity is critical, since homogeneous hierarchical bundles do not display improved properties. ${ }^{17}$ The effect of defects at the nanoscale can also be accounted for, and the HFBM has 
been used to estimate the strength and stiffness of macrostructures such as the futuristic nanotube-based space elevator cable, highlighting the defect-related decrease in performance with respect to estimations based on ideal defect-free materials. $^{18}$

Similar approaches, appropriate for two-dimensional (2D) or threedimensional (3D) simulations, are the lattice spring models (LSM) ${ }^{19}$ or random fuse models ${ }^{20}$ which provide a continuum description of the media through a network of discrete elements (springs or "resistors"). These have been used to simulate plasticity, damage propagation, and statistical distributions of "avalanches" of fracture events in heterogeneous materials ${ }^{20}$. Similar to the procedure adopted for HFBM, we recently introduced the hierarchical lattice spring model (HLSM), extending the classical LSM ${ }^{21}$ (Figure 2b). Other analytical theories such as our quantized fracture mechanics ${ }^{22}$ (QFM) or atomistic methods such as MD can be integrated in these multiscale approaches, for instance, to determine constitutive laws at the lower scale as a function of atomic structure, defect content, or molecular organization.

Other computational approaches that can also be used to model fracture propagation in a multilevel scheme include 3D meshfree models, useful for simulating complex 3D heterogeneous media with nonlocal effects. ${ }^{23,24}$ These methods have also been implemented in a multiscale scheme. ${ }^{25}$ Standard FEM approaches based on erosion algorithms are pushed to their limits when dealing with fracture nucleation and propagation, therefore, various strategies have been adopted, such as mesh adaptive refinement during the evaluation of model solutions, the use of cohesive zone model (CZM)-based elements, or extended FEM (XFEM). ${ }^{26}$ Peridynamics is a meshless method whose nonlocal response represents an ideal bridge between atomistic (MD) and continuum methods. ${ }^{27}$ This novel approach is based on a nonlocal and integral reformulation of the standard continuum theory of solid mechanics, which is applicable even when cracks and other singularities appear in the deformation field. Since peridynamics models continuous media, discrete particles, and defects using the same set of equations, it provides a means to couple different length scales. ${ }^{27}$ 


\section{Applications of modeling biomaterials}

Both theoretical models and the previously described and other numerical methods have shown that reinforcement organization in biological or bio-inspired composite materials can increase damage tolerance, avoiding direct crack path propagation and drastically improving the global response. ${ }^{28}$ Studies have focused on the influence of the structure, reinforcement shape, aspect ratio, dispersion, organization, and of mechanical properties of the constituents at various scale levels, iteratively deriving higher scale mechanical properties from lower ones, until a global material response is obtained. ${ }^{29}$

As an example, the macroscopic nonlinear (hyperelastic) constitutive properties of spider silk derived from atomistic simulations have been shown to play an essential role in the macroscopic robustness of spider webs, ${ }^{7}$ since simulations demonstrate that the same type of impact would cause more widespread damage for linear elastic and elastic-perfectly plastic counterparts. Another example of how an integrated framework can synergistically combine scalable modeling using coarse-graining and mesoscopic dissipative particle dynamics simulations for bioinspired design can be found in studies on silk spinning. ${ }^{30}$ The combined multiscale use of different computational techniques such as HFBM and HLSM has also proved to be successful in reproducing the macroscopic behavior of artificial nanocomposites such as gelatin-graphene oxide fibres. ${ }^{31}$ Mesoscale models allow the design of composite materials exhibiting tailored fracture properties, drawing inspiration from mineralized biological composites. $^{32}$

Another biomaterial property that has been studied and simulated in recent years is self-healing (Figure 2a), and its effects on the elastic, fracture, and fatigue properties of materials. ${ }^{33}$ Self-healing is incorporated in HFBM/HLSM models by replacing fractured fibers or springs with intact ones (the process of healing) having appropriate mechanical properties, volume fractions, replacement rates, and locations as damage evolves during simulations. The main control parameter is the "healing rate," defined as the ratio of the number of healed and fractured 
fibers in a given fixed time interval. "Both distributed" and "local" healing processes can be simulated, whereby fractured fibers are replaced either over the whole structure, or at concentrated locations where damage is accumulated, respectively. ${ }^{34}$ It has been found that a hierarchical structure increases the efficiency of self-healing, leading to increased toughness with respect to the corresponding nonhierarchical case and fatigue resistance, e.g. in tendons. ${ }^{35}$

The problem of simultaneous optimization of strength and toughness in materials also appears in the field of high-energy shock loadings (e.g., impacts, cutting, and blasts). Indeed, energy dissipation must occur in limited volume of material in these cases, since heavy structures are generally undesirable, such as in body armors, helmets, and crashworthy components for automotive or aerospace applications.

Extreme specific impact toughness may be pursued, for example, by the adoption of graphene-based nanocomposites ${ }^{36,37}$ or through smart bioinspired structural solutions. Dermal armors of different animals, such as the Arapaima gigas fish scales and many species of beetles, have several common characteristics, such as hard and patterned front layers, ${ }^{38,39}$ and a backing sequence of softer layers, ${ }^{38,40}$ with overall flexibility of the armor (when required for locomotion) guaranteed via discrete systems of interconnected scales..$^{38,40,41}$ All of these features are generally synergistically coupled with hierarchical assembly, which optimizes layer thickness strength and stiffness. ${ }^{38,42}$

The large variety of parameters to be considered in the study of these biological systems due to heterogeneity, the numerous levels of hierarchy, and the complexity of the constitutive laws (often strain-rate dependent) make experimental tests scarcely viable, and computational techniques are needed for the study of toughening mechanisms and for the design and optimization of bioinspired armors. Nowadays, commercial software offers robust nonlinear FEM tools for the analysis of these types of large-scale problems at an acceptable computational cost. HFBM and HLSM are useful in providing advanced constitutive response to be used as input in FEM simulations that can, therefore, be limited to details at the upper scale (Figure 2b). Thus, a series of parametric 
studies, each replicating thousands of experiments, can be performed. For example, the relationship between specific energy absorption in multilayer armors and the interface strength between layers, which could be tuned via different adhesive materials or with bioinspired hierarchical structuring of surfaces, ${ }^{43}$ has been rationalized and explained through a FEM study on composite multilayer structures. ${ }^{44}$

Modeling of impacts naturally involves friction. One of the main challenges is the multiscale modeling of hierarchical rough surfaces that emerge at the microscale or from biological optimized solutions. ${ }^{45,46}$ Explicit numerical modeling of rough surfaces features a wide range of significant length scales and would be prohibitively expensive. Because of this, a central ingredient of a multiscale approach becomes homogenization, so that macroscale friction coefficients are derived from the solution of a microscale boundary-value problem based on the smallest representative volume element (RVE) of the rough profile. ${ }^{47}$ Alternatively, isogeometric analysis, ${ }^{48}$ which exploits Computer Aided Design (CAD) splines interpolating functions both as geometry descriptors and element basis functions, is especially attractive for the analysis of complex contact geometries. This can be exploited in the analysis of armors inspired by the bombardier beetle, whose reaction chamber for the production of a defensive fluid is enclosed in undulated walls essential for greater energy dissipation and structural resistance. ${ }^{46}$

Another field in which multiscale FEM simulations are required is the modeling of dry adhesion in biological organisms, such as geckos, which is optimized using various strategies at different scale levels. Studies have shown how contact splitting (i.e., when a single contact is split into many finer ones) is used effectively by animals such as geckos and insects to increase the total peeling line (i.e. the sum of the width of the contacts)and adhesion force. ${ }^{49,50}$ This is combined at the microscale with tapering of contact units or gradation in mechanical properties to increase adhesion efficiency. ${ }^{49,51}$

Structures such as the anchorages of spider webs to external structures (Figure 2c) exploit hierarchical structure together with nonlinear constitutive 
material properties to improve resilience and to achieve tunability in adhesion/detachment characteristics. ${ }^{52}$ These mechanisms can be modeled using numerical implementations of our multiple peeling theory ${ }^{53}$ or CZM formulations within a FEM-based framework. Such computational studies have revealed how, through hierarchical structure, contact splitting and adhesion can be maximized, ${ }^{54}$ tunability achieved, ${ }^{55}$ and microcontacts optimized for improved performance. ${ }^{56}$ These results can be of great value in the design of bioinspired micropatterned adhesive surfaces. ${ }^{57}$

Another topic of interest is the study of the vibrations and wave propagation in biological or bioinspired structures, such as in the above mentioned Bombardier beetle inspired undulated structure. The role of hierarchy in the dynamic behavior of these structures remains largely unexplored to date. Studies have highlighted the possibility of enhanced vibration damping through branching, including in bioinspired slender structures. ${ }^{58}$ In the fields of phononic crystals and acoustic metamaterials (i.e., periodic structures exhibiting frequency bandgaps in which wave propagation is inhibited, or other exotic effects like focusing and cloaking), some theoretical investigations of 2D lattices with nonself-similar ${ }^{59}$ or self-similar ${ }^{60,61}$ hierarchical topologies have been performed. Preliminary numerical studies have shown that hierarchy can help create wider bandgaps at multiple frequency scales compared to simple periodic structures, ${ }^{62}$ and to tune the bandgaps and directionality of phononic crystals. ${ }^{63}$

\section{Outlook}

As seen from the discussions in this article, it appears that hierarchical design down to the nanoscale, where increased surfaces forces and material toughening mechanisms can be exploited, could be the breakthrough solution for a new generation of high-performance, multifunctional, and environmentally friendly materials. For this purpose, novel multiscale and multiphysics computational tools, such as HFBM and HLSM discussed here, need to be further developed, bridging traditional methods which otherwise cannot have an overlap scale range and enabling engineers and scientists to tailor and optimize structural mechanism in multiphase, multiscale, and multifunctional materials. To make this possible, 
the aim of computational scientists is to broaden the overlap regions in scale/time between different simulation techniques, relying on the availability of ever more powerful computational resources and, whenever possible, to enable their integration within the same computational multiscale framework.

\section{Acknowledgments}

N.M.P. is supported by the European Research Council (ERC StG Ideas 2011 BIHSNAM No. 279985 and ERC PoC 2015 SILKENE No. 693670) and by the European Commission under the Graphene FET Flagship (WP14 "Polymer Nanocomposites” No. 604391). S.S. and F.B. acknowledge support from BIHSNAM.

\section{References}

1. Z.Q. Liu, D. Jiao, M.A. Meyers and Z.F. Zhang. Acta Biomater. 17, 137 (2015).

2. H. Yao, and H. Gao. J. Mech. Phys. Solids 54, 1120 (2006).

3. C.E. Killian, R.A. Metzler, Y. Gong, T.H. Churchill, I.C. Olson, V. Trubetskoy, M.B. Christensen, J.H. Fournelle, F. De Carlo, S. Cohen, J. Mahamid, A. Scholl, A. Young, A. Doran, F.H. Wilt, S.N. Coppersmith and P.U.P.A. Gilbert. Adv. Funct. Mater. 21, 682 (2011).

4. Y. Tian, L. Jiang, MRS Bull. 40, 155 (2015).

5. M.A. Meyers, P.Y. Chen, A.Y.M. Lin and Y. Seki. Prog. Mater. Sci. 53, 1 (2008).

6. B.H. Ji, and H.J. Gao. J. Mech. Phys. Solids 52, 1963 (2004).

7. S.W. Cranford, A. Tarakanova, N.M. Pugno and M.J. Buehler. Nature 482, 72 (2012).

8. H.S. Gupta, J. Seto, W. Wagermaier, P. Zaslansky, P. Boesecke and P. Fratzl. Proc. Natl. Acad. Sci. U.S.A. 103, 17741 (2006).

9. S.W. Cranford, Biomateriomics, 1st ed. (Springer, New York, 2012).

10. S.W. Cranford, J. de Boer, C. van Blitterswijk and M.J. Buehler. Adv. Mater. 25, 802 (2013).

11. G.B. Olson. Science 277, 1237 (1997). 
12. L. Mishnaevsky, Rev. Adv. Mater. Sci. 30, 60 (2012).

13. L. Mishnaevsky and G Dai. Compos. Struct. 117, 156 (2014).

14. S. Pradhan, A. Hansen and B.K. Chakrabarti. Rev. Mod. Phys. 82, 499 (2010).

15. N.M. Pugno, F. Bosia and T. Abdalrahman. Phys. Rev. E 85, 011903 (2012).

16. L. Mishnaevsky, Compos. Sci. Technol. 71, 450 (2011).

17. F. Bosia, T. Abdalrahman and N.M. Pugno. Nanoscale 4, 1200 (2012).

18. N.M. Pugno, F. Bosia and A. Carpinteri. Small 4, 1044 (2008).

19. A.B. Gavin, M.C. Christopher and J.C. Douglas. Modell. Simul. Mater. Sci. Eng. 9, 485 (2001).

20. S. Zapperi, A. Vespignani and H.E. Stanley. Nature 388, 658 (1997).

21. L. Brely, F. Bosia and N.M. Pugno. Front. Mater. 2, 00051 (2015).

22. N.M. Pugno and R.S. Ruoff. Philos. Mag. 84, 2829 (2004).

23. S.W. Yang, P.R. Budarapu, D.R. Mahapatra, S.P.A. Bordas, G. Zi and T. Rabczuk. Comput. Mater. Sci. 96, 382 (2015).

24. E. Barbieri, and M. Meo. Compos. Sci. Technol. 69, 2169 (2009).

25. L.T. Zhang, W.K. Liu, S.F. Li, D. Qian and S. Hao. "Survey of Multi-Scale Meshfree Particle Methods," in Meshfree Methods for Partial Differential Equations, M. Griebel, M.A. Schweitzer, Eds. (Springer Berlin Heidelberg, Berlin, 2003).

26. J.H. Song, H.W. Wang and T. Belytschko. Comput. Mech. 42, 239 (2008).

27. S.A. Silling, J. Mech. Phys. Solids 48, 175 (2000).

28. D. Sen and M.J. Buehler. Sci Rep.-UK 1, 35 (2011).

29. Z.Q. Zhang, Y.W. Zhang and H.J. Gao. Proc. R. Soc. Lond.B 278, 519 (2011).

30. S.C. Lin, S. Ryu, O. Tokareva, G. Gronau, M.M. Jacobsen, W.W. Huang, D.J. Rizzo, D. Li, C. Staii, N.M. Pugno, J.Y. Wong, D.L. Kaplan and M.J. Buehler. Nat. Commun. 6, 6892 (2015).

31. S. Panzavolta, B. Bracci, C. Gualandi, M.L. Focarete, E. Treossi, K. Kouroupis-Agalou, K. Rubini, F. Bosia, L. Brely, N.M. Pugno, V. Palermo and A. Bigi. Carbon 78, 566 (2014). 
32. L.S. Dimas, G.H. Bratzel, I. Eylon and M.J. Buehler. Adv. Funct. Mater. 23, 4629 (2013).

33. A.C. Balazs, Mater. Today 10, 18 (2007).

34. F. Bosia, T. Abdalrahman and N.M. Pugno. Langmuir 30, 1123 (2014).

35. F. Bosia, M. Merlino and N. M. Pugno. J. Mat. Res., 30, 2 (2015).

36. J.-H. Lee, P.E. Loya, J. Lou and E.L. Thomas. Science 346, 1092 (2014).

37. K. Yoon, A. Ostadhossein and A.C.T. van Duin. Carbon 99, 58 (2016).

38. S.N. Patek, and R.L. Caldwell. J. Exp. Biol. 208, 3655 (2005).

39. L. Kundanati, and N. Gundiah. J. Exp. Biol. 217, 1946 (2014).

40. W. Yang, I.H. Chen, B. Gludovatz, E.A. Zimmermann, R.O. Ritchie, M.A. Meyers. Adv. Mater. 25, 31 (2013).

41. H.M. Yao, M. Dao, T. Imholt, J.M. Huang, K. Wheeler, A. Bonilla, S. Suresh and C. Ortiz. Proc. Natl. Acad. Sci. U.S.A. 107, 987 (2010).

42. R.O. Ritchie, Nat. Mater. 13, 435 (2014).

43. E. Lin, Y. Li, C. Ortiz and M.C. Boyce. J. Mech. Phys. Solids 73, 166 (2014).

44. S. Signetti, and N.M. Pugno. J. Eur. Ceram. Soc. 34, 2823 (2014).

45. M.J. Baum, A.E. Kovalev, J. Michels and S.N. Gorb. Tribol. Lett. 54, 139 (2014).

46. E.M. Arndt, W. Moore, W.-K. Lee and C. Ortiz. Science 348, 563 (2015).

47. P. Wriggers, Computational Contact Mechanics, 2nd ed. (Springer-Verlag Berlin Heidelberg, New York, 2006).

48. T.J.R. Hughes, J.A. Cottrell and Y. Bazilevs. Comput. Methods Appl. Mech. Eng. 194, 4135 (2005).

49. M. Varenberg, N.M. Pugno and S.N. Gorb. Soft Matter 6, 3269 (2010).

50. M. Kamperman, E. Kroner, A. del Campo, R.M. McMeeking and E. Arzt. Adv. Eng. Mater. 12, 335 (2010).

51. H. Peisker, J. Michels and S.N. Gorb. Nat. Commun. 4, 1661 (2013).

52. V. Sahni, J. Harris, T.A. Blackledge and A. Dhinojwala. Nat. Commun. 3, 1106 (2012). 
53. N.M. Pugno, Int. J. Fract. 171, 185 (2011).

54. F. Bosia, S. Colella, V. Mattoli, B. Mazzolai and N.M. Pugno. RSC Adv. 4, 25447 (2014).

55. L. Brely, F. Bosia and N.M. Pugno. Interface Focus 5, 20140051 (2015).

56. R.A. Sauer and M. Holl. Comput. Methods Biomech. Biomed. Eng. 16, 577 (2013).

57. G. Carbone, E. Pierro and S.N. Gorb. Soft Matter 7, 5545 (2011).

58. B. Theckes, E.d. Langre and X. Boutillon. Bioinspiration Biomimetics 6 , 046010 (2011).

59. Y.L. Xu, X.G. Tian and C.Q. Chen. Physica B 407, 1995 (2012).

60. Q.J. Lim, Wang, P., Koh, S.J.A., Khoo, E.H., Bertoldi, K. Appl. Phys. Lett. 107, 221911 (2015).

61. D. Mousanezhad, Babaee, S., Ebrahimi, H., Ghosh, R., Hamouda, A. S., Bertoldi, K., Vaziri, A. Sci. Rep. 5, 18306 (2015).

62. M. Miniaci, A. Krushynska, F.Bosia, N.M. Pugno, submitted to J. Mech. Phys. Sol. (2016).

63. D. Mousanezhad, S. Babaee, R. Ghosh, E. Mahdi, K. Bertoldi and A. Vaziri. Phys. Rev. B Condens. Matter 92, 104304 (2015).

64. E. Lepore, F. Bonaccorso, M. Bruna, F. Bosia, S. Taioli, G. Garberoglio, A.C. Ferrari and N.M. Pugno. Condens. Matter Mater. Sci. (2015), available at http://arxiv.org/abs/1504.06751.

65. M. Buehler and T. Ackbarow. Mat. Today 10, 46-58 (2007)

\section{Figure Captions}

Figure 1. Multiscale approaches to model hierarchical materials, including the region of applicability in spatial and time scales (see e.g. ${ }^{65}$ )

Figure 2. Examples of multiscale structure and simulations on the strength and toughness of hierarchical biological and bioinspired materials. (a) The mechanical behavior of nanoreinforced "bionic" silk ${ }^{64}$ is modeled using molecular dynamics simulations to derive the statistical distribution of material properties for native silk proteins mixed with carbon nanotubes or graphene at nanoscale. These distributions are then used as inputs in a Hierarchical Fibre Bundle Model, ${ }^{17}$ where hierarchical organization is modelled for the twisted strands at various hierarchical levels up to the web structure that can be modelled within a FEM framework. (b) Modeling of artificial multilayer nanocomposites inspired by 
nacre by means of a Hierarchical Lattice Spring Model $^{21}$ : results yield material constitutive laws that are fed into Finite Element Method (FEM) impact simulations $^{43}$

Figure 3. Application of the theory of multiple peeling ${ }^{55}$ and cohesive zone model (CZM) ${ }^{54}$ FEM simulations to the problem of adhesion of spider web anchorages: (a) SEM image of spider-web anchorage showing hierarchical multiple contact splitting, (b) schematization of hierarchical configuration in a multiple peeling simulation of the anchorage described by contact and split angles $(\varphi, \theta, \lambda)$ and thread length $\left(l_{\mathrm{i}}\right)$, subjected to a peeling force $F$. (c) CZM-based FEM models are used to investigate the role of fibril shape, elasticity, and multiple splitting in delamination simulations. 

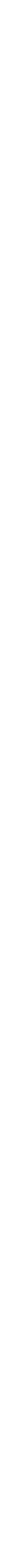